\title{
Les Amazoulous d'Abdou Anta Kâ ou la représentation tragique de la fratrie
}

\section{N'golo Aboudou SORO, Université Alassane Ouattara, Côte d'Ivoire}

Le terme fratrie tire son sens étymologique du latin frater et qui, selon Le petit Robert et Le nouveau Littré, signifie «l'ensemble des frères et sœurs d'une même famille ». La fratrie et la famille sont donc indissolublement liées et, dans Les Amazoulous du dramaturge sénégalais Abdou Anta Kâ, elles sont en dérive, voire dans un état de déliquescence avancé. Le personnage de Chaka est présenté, dans la pièce de Kâ, comme un bâtard qui a pris place sur le trône de son père au détriment des fils légitimes. En effet, le dramaturge théâtralise le roi qui, selon les historiens, a régné sur les Zoulous entre 1816 et 1828. Chaka qui serait issu d'une union illégitime entre Nandi, princesse Langeni, et Senza Ngakona, chef du clan des Zoulous est indubitablement un bâtard. Cependant, il ne s'est pas laissé attendrir par cette naissance hors mariage. Il s'est battu seul contre tous pour accéder au trône de son père. Ce «bâtard exceptionnel » est devenu par son courage, sa bravoure, son intelligence et l'ironie du sort, le guide de la multitude du peuple Ngounis qu'il a rebaptisé d'un nom qui sonne comme un tambour de guerre et tonne comme un grondement d'orage: «Zoulou », c'est-à-dire le ciel ; « Amazoulous » : ceux du ciel.

Il s'est imposé par les armes et a fait prospérer l'héritage paternel en étendant les limites du royaume au-delà des frontières insoupçonnées. Et pourtant, son père l'avait chassé de chez lui en répudiant mère et fils ${ }^{1}$. Cette réplique du chœur illustre à la fois l'origine très modeste et le génie de Chaka :

Le chœur : Amazoulous, un nom inventé par Chaka, l'enfant venu au monde, solitaie comme un soleil. Ni rythmes, ni chants ne l'annoncèrent à l'univers. Dans les herbes, il vit le jour et la honte et le mépris. (Acte I, scène 2, p.12)

Grâce à son génie militaire, Chaka a repris la place qui lui avait été ravie et désormais il est assimilé au tonnerre dont le grondement fait trembler l'homme. A cet effet, il a affirmé : «moi aussi, je regarde les peuples et ils tremblent » (Ki-Zerbo 356).

Par-delà l'héroïsme mythique de Chaka qu'elle véhicule, la pièce de Kâ permet de saisir l'accomplissement du destin glorieux d'un enfant abandonné, d'un enfant naturel ou illégitime renié par son père, pourchassé par ses demi-frères et qui a réussi à s'imposer et à imposer la trajectoire qu'il a voulu donner à l'histoire de son peuple. Cependant, une tension perdure, celle qui gouverne les relations entre Chaka et ses proches, notamment entre Chaka et Latyr, son frère consanguin ${ }^{2}$. Cette tension, avant tout politique, parcourt toute l'œuvre de Kâ 
et imprime à l'action dramatique un caractère tragique. Ce qui justifie la thématique : «Les Amazoulous de Kâ ou la représentation tragique de la fratrie ».

L'œuvre donne l'occasion au dramaturge de mettre sur les planches la rivalité entre deux demi-frères nés d'un père polygame. Cela pose la problématique de l'héritage dans la famille africaine où, très souvent, sous l'influence des épouses, il s'établit une discrimination négative et nocive entre les enfants. L'objectif de cette étude est d'élucider la façon dont Kâ dramatise la rivalité fratricide qui a abouti à l'assassinat de Chaka. Par quels moyens les antagonismes fratricides entre Chaka et Latyr sont-ils mis sur scène ? Quelle est la portée idéologique de la tragédie fratricide de Kâ?

\section{I - Chaka et Latyr : une fratrie déchirée}

La fratrie, dans Les Amazoulous, se résume à deux frères consanguins : Chaka et Latyr. Mais ce sont deux frères ennemis, tant Latyr en veut à Chaka de l'avoir évincé du trône laissé par leur défunt père. Dans cette pièce qui s'inspire de l'histoire du héros zoulou, la fratrie a été élaguée et, de trois demi-frères que les historiens évoquent dans leurs écrits, l'auteur ne nous présente que deux dont l'un, Latyr, est défiguré pour montrer l'ignominie de son acte. En effet, l'auteur du fratricide apparaît dans l'œuvre dramatique de Kâ sous un nom fictif. Mais qui sont ces demi-frères antipathiques sur la scène Antaenne?

\section{I-1- Chaka : le bâtard au destin mythique}

Dans Les Amazoulous, la fable est construite sur le modèle de l'analepse. Cela permet la représentation des réminiscences des personnages à partir desquelles le lecteur-spectateur s'efforce de construire la vie de Chaka. A travers les discours des autres personnages et de Chaka lui-même, l'on découvre ses origines modestes, son enfance difficile, son règne et sa fin malheureuse. Dès la scène II de l'Acte I, nous savons avec le chœur que Chaka a connu le reniement paternel avant même sa naissance, ainsi que le rejet de la part de ses frères. Suivons à ce propos le discours du Chœur :

LE CHCEUR : Renié par son père, haï par ses frères !... Chaka le bâtard ! ...

LE CHCER : Et lui Chaka, chaque dent l'éloignait davantage de son village ; dent après dent, sa mort ordonnée par son père, le menait toujours plus loin... Chaka survécut, mangeant ce qu'il trouvait. Parfois, des journées entières, à demi-mort de faim, la vision des âmes fortes le soutenait dans sa longue marche. (Acte I, scène 2)

L’image métonymique que charrie le syntagme « chaque dent l'éloignait » indique que, dès son jeune âge, Chaka a été contraint de s'exiler pour échapper à la mort. Par-delà l'exil, la mère devait continument fuir avec son fils pour échapper aux bourreaux lancé à leurs trousses. 
Par ailleurs, les affres de cet exil du prince renié et condamné transparaissent dans les hyperboles : «mangeant ce qu'il trouvait », « demi-mort » et «journées entières ». Toute cette rhétorique d'amplification permet au dramaturge de traduire de façon ostensible, les privations et les souffrances endurées par Chaka pendant la fameuse période de disgrâce. Il a connu une enfance pénible et errante. A peine sorti du berceau, le héros des Amazoulous de Kâ est livré aux vicissitudes d'une enfance sans autorité paternelle et exclusivement à la charge de sa mère. Le mot est lâché par le chœur et il parcourt tout le texte théâtral: Chaka est un bâtard. L'exclamation «Chaka le bâtard!» traduit, ici, à la fois l'étonnement et l'indignation. Le héros de Kâ, en dépit du sang royal qui coule dans ses veines, se voit éloigner du patrimoine paternel. Avec lui, l'on assiste à la réactualisation d'une variante de l'hostilité œedipienne. Comme le héros tragique de l'Antiquité, Chaka a été contraint de vivre loin de son père et du palais où il est venu s'installer après la mort de celui-ci. Aussi, peut-il être présenté comme une métaphore de l'enfant de la rue, dans l'Afrique moderne, qui a bravé les obstacles de la vie et a réussi à se forger un destin glorieux. Chaka a bravé toutes les adversités artificielles et naturelles liées à cette vie de paria social.

Cependant, il ne s'offusque pas de cette enfance laborieuse faite de frustration et de privation. Il s'en sert plutôt pour donner force à sa parole. A la fin de la tirade à la page 52, il proclame solennellement qu': «il n'y aura plus d'hommes inférieurs dans l'empire des Amazoulous, je vous en donne ma parole, moi Chaka, l'enfant né dans les champs » (Acte I, scène III). Ce qui devait constituer pour lui une honte ou une indignation est évoqué avec l'intention bienheureuse de défendre les plus faibles contre toutes formes de domination arbitraire. Pour avoir subi l'injustice, il s'érige en justicier afin de protéger les plus faibles. Cependant, son demi-frère, Latyr, évoque avec morgue et de façon désinvolte la triste situation de Chaka au sein de la fratrie afin de nuire à son image, à son honorabilité, et surtout dans le but de le discréditer auprès des autres rois. En témoigne, sa conversation avec le vieux roi :

LE VIEUX ROI : Votre couronne ! L'auriez-vous perdue en cours de route ? LATYR : aujourd'hui sur la tête d'un bâtard.

LE VIEUX ROI : Les couronnes et les bâtards... des fiancés de longue date...Chaka est donc un bâtard exceptionnel...

LATYR : Un bâtard exceptionnel! Un mendiant, un misérable vagabond, la boue et la bave. Il se disputait la charogne des animaux avec les vautours, nourri d'une rage : tuer, encore tuer, allant même jusqu'à se battre avec son ombre. Un bâtard exceptionnel ? Un bâtard, un malvenu, une vermine. (Acte I, scène III, 50)

La question rhétorique et la gradation ascendante qui achèvent la tirade attestent que pour Latyr, Chaka ne mérite pas le trône dans la mesure où sa place est remise en cause dans la famille royale. Les injures qu'il profère à son endroit sont révélatrices de l'hostilité qui règne 
au sein de la fratrie et de la déconfiture de celle-ci dans la pièce de Kâ. Le frère du héros lui voue une haine implacable. Latyr s'en prend vertement à Chaka à travers invectives et diffamations. Chaka ou «le bâtard exceptionnel », comme l'indique le vieux roi, n'est pas accepté par son frère comme tel. Il est plutôt vu comme un usurpateur notoire.

La conception de Chaka, qui, selon les historiens, s'est faite hors mariage, pèse sur sa vie et annonce une rupture dans la fratrie. Cependant, celui qui est sorti de nulle part parce que non reconnu par son père et «qui ne savait où aller, pourchassé comme un lépreux par son clan » (Acte I, Scène 1,43) est présenté dans le texte théâtral comme un fondateur d'empire. Les autres personnages, à l'exception de Latyr et des prêtres, lui reconnaissent des qualités de chef charismatique et l'admirent. Avec lui, le peuple est protégé et vit en paix. Par la mise en scène d'un Chaka conquérant et défenseur des plus faibles, le dramaturge reste collé à la vérité historique selon laquelle «Chaka est un des grands de l'Afrique australe et son nom mérite d'être retenu par l'histoire universelle » (Ki-Zerbo 360). Cependant, son demi-frère Latyr, qui refuse d'accepter cette noblesse, opte pour le dénigrement et le complot.

\section{I -2- Latyr, le demi-frère ennemi}

Le personnage de Latyr échappe à l'histoire, car défiguré. Il semble avoir perdu son identité historique pour devenir un personnage fictif inventé par le dramaturge. En effet, comme le dit Bassidiki Kamagaté, «si dans Les Amazoulous, Chaka et Nolivé renvoient à des personnages historiquement réels, bon nombre de personnages ont été défigurés, voire rebaptisés. Ceux qui ont souffert de leur perte d'identité restent les assassins de Chaka comme pour attester l'ignominie de leur acte » (119).

Cependant, par ses agissements, l'on reconnaît, sous les traits de Latyr, les frères consanguins historiquement réels que sont Din'ngana et Malha'ngan. Le dramaturge, par son génie créateur et pour faire de la place à la fiction théâtrale, a fusionné les deux demi-frères en un unique demi-frère à Chaka. Un demi-frère qui se présente comme le fils légitime à qui revient la couronne des N'gounis. Aussi, qualifie-t-il Chaka de «bâtard », de «malvenu » et de « vermine ». Ce discours ignoble et digne de l'ennemi est caractéristique de la personnalité de Latyr : une personnalité obsédée et grisée par sa naissance royale annoncée à bruits de tamtams et de balafons et qui « fut reprise en chœur par tous les cors du royaume » (Acte I, scène $\mathrm{V}, 57)$. C'est justement cette naissance princière qui l'aveugle et l'empêche de voir que sous le règne de Chaka, le peuple vit dans l'opulence comme l'indique le vieux roi à la scène IV, de 1'Acte I, pp.54-55. 
Le fils légitime en veut mortellement à son demi-frère à qui il reproche d'usurper son trône, tant sa naissance fut anonyme et son enfance malheureuse. Dans la réplique qui suit, Latyr se déchaîne contre son frère :

LATYR : L'héritier est devenu le paria. Quel combat que le nôtre, Chaka, toi et tes semblables. Vous sortez des dépotoirs, l'empreinte d'aucune famille sur le front, étrangers dans la cité, tant vos haillons portent ailleurs nos regards. (Acte I, scène $V, 57$ )

Le discours à la fois hâbleur et réducteur de Latyr rompt ici avec les valeurs chevaleresques dignes d'un prince héritier. En se fondant sur des considérations qui ne prennent pas en compte les qualités intrinsèques de Chaka, son frère tombe dans le camp des fils à papa à qui la seule valeur est de pouvoir se vanter d'une naissance fortunée. Pour Latyr, les circonstances de sa naissance suffisent pour faire de lui un roi. Mais, comme le dit Egbuna Modum dans son article «Le mythe de Chaka», publié dans le numéro14 de la revue Éthiopiques, «Gilbert Durand a raison de dire que le fils à papa ne peut jamais devenir un héros mythique, mais restera l'éternel médiocre, condamné à l'état de banalité ».

À travers la mise en scène d'un fils chef lequel seule sa « légitimité » le fait croire à son droit au trône, Kâ tourne en dérision les politiques légitimistes qui ne prennent nullement en compte la valeur intrinsèque du prétendant. Le dramaturge trouve ici le moyen artistique de dénoncer la mainmise de certains dirigeants africains sur la politique de leur État, notamment de ceux qui tentent de créer des dynasties ou des oligarchies qui excluent les autres, jugés de mauvaises familles. Latyr symbolise justement, le fils à papa qui n'a jamais appris à affronter la réalité comme l'enfant de la rue ou l'enfant aux parents démunis, inconnus de la sphère des gouvernants et qui se sent obligé d'accomplir des exploits surhumains afin de mériter sa place dans la société. Tel fut le cas de Chaka, car rejeté par son père et livré à lui-même. Latyr se présente comme le demi-frère-ennemi-mortel de Chaka. Il lui voue une haine implacable au point de le dénigrer, de le vilipender et même de comploter contre lui dans le but de l'évincer du trône. D'où la tragédie au sein de la fratrie.

\section{II- Les antagonismes fratricides, sources de tragédie dans Les Amazoulous}

La fratrie dans Les Amazoulous du dramaturge sénégalais est sujette à des antagonismes générateurs de tragédie. Le héros de Kâ est précipité dans l'abîme tragique, non du fait d'une quelconque volonté divine mais, du fait de son rejet par son frère et surtout du désir effréné de celui-ci d'être roi. La fin tragique de Chaka est donc le fait de la combinaison de variétés d'effets tragiques parmi lesquels figurent en bonne place la politique et la rivalité fratricide. 


\section{II-1- La rivalité fratricide et le fait tragique}

La rivalité créée et entretenue par Latyr qui, se prévalant d'une légitimité de naissance, n'admet pas que Chaka hérite du trône d'un père qui l'a chassé de son vivant. A partir de ces considérations dignes d'un aristocrate arriviste, le demi-frère de Chaka se ligue contre lui dans le but ultime de prendre sa place. La mise à exécution de ce plan machiavélique est l'une des sources de la tragédie dans Les Amazoulous de Kâ. En effet, comme le dit Pierre Corneille dans son Discours de la tragédie : «Qu'un ennemi tue ou veuille tuer son ennemi, cela ne produit aucune commisération [...] qu'un indifférent tue un indifférent, cela ne touche guère davantage [...] ; mais quand les choses arrivent entre des gens que la naissance ou l'affection attache aux intérêts l'un de l'autre, comme alors qu'un mari tue ou est prêt de tuer sa femme, une mère ses enfants, un frère sa sœur, c'est ce qui convient merveilleusement à la tragédie » (95).

La pièce de Kâ exploite ce versant de la tragédie. Latyr porte le coup fatal à son frère. Il est aidé dans sa besogne par Kaïn :

LE PREMIER HOMME MASQUE (désignant Chaka) : Latyr, cet homme était ton demi-frère.

LATYR : J'ai frappé le premier.

LES TROIS HOMMES MASQUES : Kaïn.

KAÏN : (s'avance, saisi d'un tremblement de tout son être et désignant Chaka) :

Il fut mon ami.

LES TROIS HOMMES MASQUES (à la fois) : Les faits ! Les faits !

KAÏN : J'ai frappé le second. (Le poignard sculpté tombe des dessous de son habit.) Avec ce poignard ! Avec ce poignard !

NOLIVE : Ton poignard, Chaka. (Acte I, scène I, 40)

Latyr et Kaïn sont les auteurs d'un fratricide dans le sens qu'en donne Valy Sidibé dans son ouvrage Le tragique dans le théâtre de Bernard Binlin Dadié, c'est-à-dire d'un «crime contre non seulement son père, mais contre tout être à qui l'on est lié par nature, tout crime dénaturé » (13). Dans une parodie de jugement, le dramaturge dévoile le tragique fratricide non sans condamner l'acte ignoble de Latyr et de Kaïn. Chez Kaïn, l'exclamation et la répétition, « Avec ce poignard ! Avec ce poignard ! », dévoilent une double trahison : non seulement il est ami à Chaka, mais il s'est servi du poignard de son ami pour l'assassiner. D'où le fait d'être saisi par un tremblement à la vue de Chaka. La didascalie intégrée qui indique que «le poignard sculpté tombe des dessous de son habit» permet au dramaturge de montrer la forfaiture dont s'est rendu coupable Kaïn. La motivation de Kaïn se découvre à la scène 1 de l'Acte III qui s'ouvre avec «Chaka en habit impérial sur le trône ». Parlant de Chaka, Kaïn se confie au dignitaire Assouk : 
KAÏN (à Assouk) : Vénérable Assouk, connais-tu la première qualité des grands de ce monde : la mise en scène. Il va nous servir ses visions. (Acte III, scène 1, 71)

Il se lit dans cet aparté de l'ami de Chaka une sorte de lassitude à l'écouter et à exécuter des ordres sans jamais être consulté. L'adjectif possessif «ses » qui, avec toute la charge hyperbolique conséquente, détermine le substantif «visions» et le verbe métaphorique «servir » qui le précède dans la dernière phrase de la réplique nous renseignent sur la qualité des relations qui lie les deux amis. Kaïn ne supporte plus d'être méprisé par Chaka du fait de son rang social. Les propos de Kaïn révèlent son amertume causée par l'envie d'être à la place de Chaka ou tout au moins d'être consulté comme un ami.

$\mathrm{Si}$, ce personnage théâtral de Kâ est une transposition de Mbopa, l'ami historique de Chaka qui s'est joint à ses frères pour l'assassiner, il rappelle aussi le personnage biblique de Caïn qui a tué son frère Abel par jalousie. A travers ce nom-masque, le dramaturge sénégalais fait ressurgir un «personnage déjà codé », pour se servir d'une expression de la critique Anne Ubersfeld dans son texte Lire le théâtre (137). Cela lui permet de mettre davantage en exergue le tragique des rivalités fratricides et par la même occasion de faire entrer Chaka dans la catégorie des héros de la trempe d'Abel, le frère jumeau de Caïn dans le récit biblique. Chaka accède ainsi à la pyramide des Héros de la race quand son frère s'engouffre avec Kaïn dans l'empire de la boue.

\section{II-2- La politique, source du tragique dans Les Amazoulous d'Abdou Anta Kâ}

La politique, comme le dit Sidibé, «reste essentiellement tragique quel que soit son mode d'orientation » (22). La parodie de procès qui porte sur les motivations de l'assassinat de Chaka permet de mettre à nu l'aspect sordide de la politique, ce que Michel Prigent dénomme le «masque de l'intérêt, de l'ambition, de l'amour propre » dans le théâtre de Corneille (153). Les antagonismes consubstantiels au choc des ambitions au sein de la fratrie sont à l'origine de la mort du héros zoulou. Dans la pièce, Chaka n'a pas été assassiné pour ses atrocités, ou ses dérives dictatoriales dont l'histoire coloniale l'accuse. Latyr l'a assassiné dans le seul but de prendre sa place sur le trône de leur défunt père. Son orgueil princier à commander aux autres ne s'est jamais accommodé avec son statut subordonné à celui qu'il trouve indigne du trône.

Le reniement paternel et l'enfance malheureuse militent en la défaveur du héros au sein de sa fratrie : Latyr et ses alliés sont grisés par l'idée de perpétuer une dynastie monarchique qui exclut Chaka; ils tentent donc de rallier à leur cause le vieux roi de l'est. A travers la dramatisation de cette tentative d'ourdir un complot depuis l'extérieur et notamment à partir d'un royaume frontalier, le dramaturge lève le voile sur la façon dont certains opposants politiques africains procèdent pour attenter à la sureté de leurs pays. 
Cependant, le roi contacté par Latyr refuse de se liguer contre Chaka qu'il admire. La scène IV de l'Acte III s'ouvre sur la suite du procès avec le témoignage du vieux roi de l'est qui n'a pas voulu tremper dans le complot parce qu'il considère Chaka comme le «saint » qui sauva son peuple :

LE VIEUX ROI : ... Honorables Dieux, les greniers se vidaient; les vaches vêlaient. Que restait-il à mon peuple ? Trouver un saint pour leur faire manger des cailloux. Y avait-il un saint parmi tes prêtres?

LATYR : Midi, midi s'annonçait victorieux.

LE VIEUX ROI: Midi, midi, encore de la poésie. De l'autre côté des montagnes, le peuple entier de Chaka mangeait à sa faim. Mes montagnes ne peuvent pas faire taire les langues. (Acte III, scène IV, 55)

Ce dialogue entre le vieux roi et Latyr est révélateur de la paix et de l'abondance dans lesquels vivaient les peuples sous l'empire de Chaka. La métaphore et toute la charge laudative que renferment le terme «saint» par lequel Chaka est désigné et les questions rhétoriques qui s'accumulent dans les propos du vieux roi montrent le rôle prépondérant du roi zoulou dans l'avènement d'une société paisible. Il est à l'origine de l'émergence d'une société où l'autosuffisance alimentaire est une réalité tangible. Le roi ne comprend donc pas les raisons du complot qui n'aura pour conséquence que de priver le peuple de « celui qui vivifie les âmes, les refleurit de toutes couleurs » (Acte I, scène II, 42). C'est en fait ce vieux tombé sous le charme de Chaka qui l'a baptisé «bâtard exceptionnel ». Il a rejeté les propositions perfides et funestes de Latyr et témoigne contre lui dans cette mise en scène du jugement dernier. Latyr refuse de se laisser attendrir par les arguments du vieux monarque. En revanche, il s'est donné les moyens d'attenter à l'intégrité physique de son demi-frère. Par ce geste hautement tragique, abject et digne du félon qu'il incarne, Latyr s'est définitivement engouffré dans la boue de l'histoire comme tous ces faiseurs de coups d'Etat qui ont longtemps terni l'image de l'Afrique. C'est ce qui, selon Kamagaté dans l'article « De l'histoire au théâtre historique dans Les Amazoulous d'Abdou Anta Kâ » (119), justifie la perte de son identité historique. Kâ a réinventé l'assassin du roi zoulou et au-delà de son acte infâme, son discours permet de saisir des problèmes d'actualité tant au plan politique que social. Ainsi, au-delà de la politique, la dramatisation des antagonismes entre Chaka et Latyr actualise la question de la ségrégation au sein de la fratrie dans une famille polygame africaine.

\section{III- Une réflexion sur la discrimination au sein de la fratrie}

Dans Les Amazoulous la constitution de la fratrie s'est faite avec en son sein des germes confligènes. Étant demi-frères, ce qui signifie que les liens de parentés entre Chaka et Latyr se situent autour d'un seul parent, en l'occurrence le père. Quant aux mères, elles sont des rivales. 
Selon les historiens, les autres épouses de Senza Ngakona $^{3}$ vont l'amener à bouter hors du foyer conjugal la mère de Chaka avec son fils. Se fondant sur cette position tranchée et partisane de leur père, Latyr considère son demi-frère Chaka comme un bâtard qui ne mérite pas de siéger sur le trône des Zoulous. Cela pose le problème de la responsabilité du père d'une fratrie constituée de demi-frères.

La pièce traduit littérairement la discrimination dont le personnage historique de Chaka a été victime de la part de son père et son rejet par la fratrie. Non seulement, son père l'a renié et l'a chassé avec sa mère, mais ses frères ne l'ont jamais accepté comme tel. Latyr a mis tout en œuvre pour l'assassiner afin d'accaparer le trône laissé par leur père. Cette personnalité tragique de Latyr a été forgée par l'attitude du père dans cette famille royale africaine ou cohabitent plusieurs épouses. Les enfants nés des nombreuses épouses ne bénéficient pas toujours de la même estime de la part du père qui, lui, subit très souvent l'influence de chacune de ses épouses. Cela n'est pas sans conséquence sur l'éducation des enfants avec pour corollaire la marginalisation et l'abandon. La ségrégation de la part du père ou des mères au sein de la fratrie débouche inéluctablement sur des frustrations et des conflits. En revanche, bien entretenues, les relations fraternelles favorisent la socialisation de l'individu.

Pour Bourguigon que cite Monique Buisson dans son livre La fratrie, creuset des paradoxes, la fratrie offre «l'expérience concrète et vécue du socius, impliquant une découverte et une utilisation toujours conscientes des stratégies qui permettent la vie commune et le développement individuel »(21). Cependant, cela n'est pas toujours valable pour une fratrie où l'on met au premier plan les différences entre les enfants. En cultivant les dissemblances entre eux, en préférant l'un à l'autre, les parents cristallisent très souvent et rarement de façon consciente la rivalité et la jalousie consubstantielles à la fratrie qui dès lors cesse d'être le creuset fondateur dans lequel on apprend à « se frotter » à l'autre, où les frères et les sœurs apprennent à se différencier et à comprendre que chacun peut avoir son territoire sans empiéter sur celui de l'autre. Dans ce cas, «Les relations fraternelles, mises en scène dans un mode binaire, opposent deux frères pour la lutte au pouvoir» (Buisson, 13) ou la reconnaissance. Les récits de la mythologie classique, les tragédies antiques et les écritures bibliques évoquent des antagonismes fratricides de cet ordre et les exemples sont légions.

Dans Les Amazoulous Chaka et Latyr n'échappent pas aux effets pervers de cette discrimination. Ils ont grandi séparément, ignorant même qu'ils sont frères au point que Latyr remet en cause la légitimé de Chaka au moment où intervient le choc des intérêts et que Chaka accapare le trône de leur père. Pour eux donc, la fratrie n'a jamais été le lieu idéal pour apprendre à gérer les conflits ou à s'attacher l'un à l'autre. 


\section{Conclusion}

La fratrie dans Les Amazoulous de Kâ est constituée de deux demi-frères dont l'un est considéré comme illégitime. Latyr est conforté dans sa position de rejet de la légitimité du chef des Zoulous par le fait que leur père avait de son vivant renié et chassé Chaka et sa mère du palais royal. Avec cette pièce, le dramaturge théâtralise une fratrie en déliquescence et dont les antagonismes sont sources de tragédie ; Latyr finit par tuer Chaka. Grâce à l'analepse, Kâ nous le présente au début de la pièce avouant son crime sous la menace de trois personnages masqués. Le demi-frère parricide a eu le soutien d'un homme très proche de Chaka, son ami Kaïn. Le nom de ce personnage rappelle bien le Caïn parricide de la Bible qui, jaloux de son frère Abel, l'a tué. L'analogie onomastique entre le personnage dramatique de Kâ et le personnage biblique et la contiguïté historique des gestes par eux accomplis, participent de la volonté du dramaturge de montrer que la malédiction accompagne inéluctablement le fratricide. La fiction théâtrale permet au dramaturge de faire ce rapprochement afin de mettre en exergue les travers de la désunion au sein d'une fratrie.

Au-delà de la théâtralisation de l'histoire du héros zoulou et de la fratrie abîmée, le dramaturge semble s'insurger contre la discrimination entre les enfants dans une famille polygame ou même au sein d'une famille. Les Amazoulous est une pièce actuelle dans la mesure où le phénomène de demi-frères est bien réel dans la société sénégalaise et en Afrique noire. Il s'agit d'une occasion pour le dramaturge d'attirer l'attention du lecteur-spectateur sur les travers d'une quelconque discrimination au sein de cette fratrie délicate qui ne tient que par un parent et est susceptible d'éclater à la moindre contrariété en son sein.

\section{Bibliographie}

Buisson, Monique. La fratrie, creuset des paradoxes. Paris : Harmattan, 2003.

Camdessus, Brigitte. La fratrie méconnue : liens du sang, liens du cæur. Paris : ESF, 1998.

Corneille, Pierre. Discours de la tragédie. Paris : La Pléiade, I.

Kâ, Abdou Anta. Les Amazoulous. Théâtre, auatre pièces d'Abdou Anta Kâ. Paris : Présence Africaine, 1972. 35-75.

Kamagaté, Bassidiki. «De l'histoire au théâtre historique dans Les Amazoulous d'Abdou Anta Kâ ». Études françaises 45-3 (2009).

Kesteloot, Lilyan et Bassirou Dieng. Les épopées d'Afrique noire. Paris : Karhala et UNESCO, 2009.

Ki-Zerbo, Joseph. L'histoire de l'Afrique noire d'hier à demain. Paris : Hatier, 1978. 
Lugan, Bernard. Histoire de l'Afrique du Sud de l'Antiquité à nos jours. Paris : Perrin, 1990.

Modum, Egbuna. «Le mythe de Chaka ». Éthiopiques 14. Revue socialiste de culture négroafricaine (avril 1978). http://ethiopiques.refer.sn/spip.php?article647, consulté le $4 / 06 / 201$.

Prigent, Michel. Le héros et l'Etat dans la tragédie de Pierre Corneille. Paris : PUF, 1986.

Sidibé, Valy. «Le mythe de Chaka dans la littérature dramatique négro-africaine ». Maestri Temi e Correnti : Impronte nelle letterature extra-europe. Bulzoni. 101-110.

Ubersfeld, Anne. Lire le théâtre. Paris : Éditions sociales, 1982.

\section{NOTES}

${ }^{1}$ Dans les livres II et III de Chaka le Grand (1979), Mazisi Kumene fait part du rejet de Chaka par son père et des malheurs de l'exil auquel il a été contraint avec sa mère. Cf. Lilyan Kesteloot et de Bassirou Dieng (578).

${ }^{2}$ Selon Monique Buisson, «lorsqu'ils ont en commun leur seul père, ils sont frères et sœurs consanguins... » Cf. note infra pages 17-18. La fratrie creuset des paradoxes.

${ }^{3}$ Chaka est le fils de Senza Ngakona, chef de clan Abatetwa (une fraction du peuple Ngouni) en Afrique du Sud. 